\title{
Agrobacterium spp. nosocomial outbreak assessment using rapid MALDI-TOF MS based typing, confirmed by whole genome sequencing
}

Carlo Casanova ${ }^{1 *+} \mathbb{D}$, Elia Lo Priore ${ }^{2 \dagger}$, Adrian Egli ${ }^{3,4}$, Helena M. B. Seth-Smith ${ }^{3,4}$, Lorenz Räber ${ }^{5}$, Daniel Ott ${ }^{6}$, Valentin Pflüger ${ }^{7}$, Sara Droz ${ }^{1}$, Jonas Marschall ${ }^{2}$ and Rami Sommerstein ${ }^{2^{*}}$

\begin{abstract}
Background: A number of episodes of nosocomial Agrobacterium spp. bacteremia (two cases per year) were observed at Bern University Hospital, Switzerland, from 2015 to 2017. This triggered an outbreak investigation.

Methods: Cases of Agrobacterium spp. bacteremias that occurred between August 2011 and February 2017 were investigated employing line lists, environmental sampling, rapid protein- (MALDI-TOF MS), and genomebased typing (pulsed field gel electrophoresis and whole genome sequencing) of the clinical isolates.

Results: We describe a total of eight bacteremia episodes due to A. radiobacter $(n=2)$, Agrobacterium genomovar G3 $(n=5)$ and A. pusense $(n=1)$. Two tight clusters were observed by WGS typing, representing the two $A$. radiobacter isolates (cluster I, isolated in 2015) and four of the Agrobacterium genomovar G3 isolates (cluster II, isolated in 2016 and 2017), suggesting two different point sources. The epidemiological investigations revealed two computer tomography $(\mathrm{CT})$ rooms as common patient locations, which correlated with the two outbreak clusters. MALDI-TOF MS permitted faster evaluation of strain relatedness than DNA-based methods. High resolution WGS-based typing confirmed the MALDI-TOF MS clustering.

Conclusions: We report clinical and epidemiological characteristics of two outbreak clusters with Agrobacterium. spp. bacteremia likely acquired during $\mathrm{CT}$ contrast medium injection and highlight the use of MALDI-TOF MS as a rapid tool to assess relatedness of rare gram-negative pathogens in an outbreak investigation.
\end{abstract}

Keywords: Agrobacterium, Rhizobium, Nosocomial outbreak, MALDI-TOF MS, Whole genome sequencing

\section{Background}

Bacteria of the genus Agrobacterium are environmental gram-negative rods, present in soil and plants. They are rare opportunistic human pathogens, with the majority of infections reported in immunocompromised hosts, such as oncology patients, the elderly, and neonates [1-3]. Central venous catheter (CVC)-related bloodstream infection (BSI) is the most common clinical presentation and is

\footnotetext{
* Correspondence: carlo.casanova@ifik.unibe.ch; rami.sommerstein@insel.ch ${ }^{+}$Carlo Casanova and Elia Lo Priore contributed equally to this work. ${ }^{1}$ Institute for Infectious Diseases, University of Bern, Friedbühlstrasse 51, 3001 Bern, Switzerland

${ }^{2}$ Department of Infectious Diseases, Bern University Hospital, University of Bern, Freiburgstrasse, 3001 Bern, Switzerland

Full list of author information is available at the end of the article
}

occasionally preceded by exposure to soil $[4,5]$. Other clinical manifestations include spondylodiscitis, endophthalmitis, pneumonia, peritonitis, urinary tract infection and endocarditis [6-11]. Furthermore, an Agrobacterium strain has been recognized as a cause of pseudobacteremia following contamination of blood cultures in the microbiology laboratory [12]. Although nosocomial cases have been described before, a common source has never been reported $[8,11]$. Recently, A. radiobacter strains were reported in a three-species outbreak in Brazil associated with total parenteral nutrition [13].

The taxonomy and nomenclature of the genus Agrobacterium has been subject of a long debate $[14,15]$ but has now come to a consensus [16-19]. To date, at least

(c) The Author(s). 2019 Open Access This article is distributed under the terms of the Creative Commons Attribution 4.0 International License (http://creativecommons.org/licenses/by/4.0/), which permits unrestricted use, distribution, and reproduction in any medium, provided you give appropriate credit to the original author(s) and the source, provide a link to the Creative Commons license, and indicate if changes were made. The Creative Commons Public Domain Dedication waiver (http://creativecommons.org/publicdomain/zero/1.0/) applies to the data made available in this article, unless otherwise stated. 
20 genomospecies are recognized [20] whereof many are yet to receive valid latin binomial names. Notably, a number of previous clinical reports on strains of "Rhizobium radiobacter" and "Agrobacterium tumefaciens" point at the same species, now officially termed Agrobacterium radiobacter (= genomovar G4 of the " $A$. tumefaciens species complex") [19].

A variety of molecular techniques are available to determine relatedness of bacterial isolates in outbreak situations [21]. Pulsed-field gel electrophoresis (PFGE) has been considered the "gold standard" typing method for many bacterial species; however, the method is not easily transferable between laboratories and requires a high level of standardization. Next-generation sequencing technologies are currently becoming more widely available for medical microbiology laboratories and typing by analysis of whole genome sequencing (WGS) data is the method offering the highest discriminatory power [22]. The introduction of MALDI-TOF MS has revolutionized clinical bacteriology and is used in many laboratories for rapid identification of most clinically relevant bacterial species at very low cost. Beyond species identification, the utility of MALDI-TOF MS-based typing for outbreak investigation has been demonstrated for several pathogens, including Acinetobacter baumannii, Enterobacter cloacae and Escherichia coli [23-28]. For Enterococcus faecium and Staphylococcus aureus, however, it is more controversially discussed whether the discriminatory power of MALDI-TOF MS is sufficient for strain typing $[24,29,30]$, indicating that the applicability of this technique may depend on the bacterial species. Here, we investigated a series of nosocomial Agrobacterium spp. bacteremia episodes observed at Bern University Hospital, Switzerland, and used MALDI-TOF MS-based typing as first-line method to determine relatedness of the isolates, which was subsequently confirmed by highresolution WGS-based typing.

\section{The event (index cases)}

Index case A was a 72-year-old male admitted on November 26, 2015 for an elective transcatheter aortic valve implantation. A computed tomography (CT)angiography was performed on the admission day in $\mathrm{CT}$ room 1 . The next day, the patient underwent diagnostic cardiac catheterization in the catheter laboratory A, followed by transcatheter aortic valve implantation in the hybrid procedure room. Three days later he developed fever with rigors, an elevated C-reactive protein (CRP) of $147 \mathrm{mg} / \mathrm{l}$, and a leucocyte count of $15.0 \mathrm{G} / \mathrm{L}$. At time of symptom onset, the left internal jugular CVC and a percutaneous sheath introducer, both inserted upon hospital admission, had already been removed. No alternative focus of infection was found. Both peripherally drawn blood cultures revealed growth of $A$. radiobacter. The tip culture of the removed CVC showed no growth. Antimicrobial treatment included empirical intravenous amoxicillin-clavulanate, cefepime and later ceftriaxone for a total duration of 10 days. The clinical course was favorable (Additional file 1: Figure S1).

Index case B was an 83-year-old female admitted on December 1, 2015 with acute congestive heart failure in the setting of severe aortic stenosis. She underwent diagnostic cardiac catheterization in catheter laboratory A on the same day. CT-angiography was performed 2 days later in CT room 1. On day three after catheterization she developed fevers with chills accompanied by an elevated CRP of $19 \mathrm{mg} / \mathrm{l}$ and a leucocyte count of $12.5 \mathrm{G} / \mathrm{L}$. The only vascular access present was a peripheral venous catheter on the left forearm inserted upon hospital admission. No other potential focus of infection was detected. Both blood cultures drawn were positive for $A$. radiobacter. The peripheral catheter tip was not cultured. The patient was empirically treated with intravenous amoxicillin-clavulanate, followed by meropenem plus ciprofloxacin for a total duration of 14days. The clinical course was favorable (Additional file 1: Figure S1).

These two index cases triggered the outbreak investigation.

\section{Methods \\ Epidemiological and clinical investigation}

A case was defined as a patient with an Agrobacterium or Rhizobium sp. isolate from at least one blood culture during the hospital stay at Bern University Hospital, a 950-bed tertiary care center. Potential cases were reported by the infectious diseases consultation service and/or identified via a structured query of the microbiology laboratory's database with the search criteria ["Agrobacterium" OR "Rhizobium"] AND "blood culture" in the time period from 01 January 2011 to 31 December 2017. We then collected data on clinical and epidemiological baseline characteristics of the cases as well as treatment and outcome data.

We generated a line list in order to identify possible common sources. This was followed by on-site audits of identified common locations and environmental swabs of suspected sources. Environmental sampling was performed with premoistened swabs, cultured on MacConkey agar and CHROMagar ${ }^{\text {Ti }}$ Orientation (Becton Dickinson, Sparks, USA) (Additional file 1: Table S1).

\section{Identification of blood culture isolates}

Blood cultures were taken according to clinical routine and only if clinically indicated. Inoculated BacT/ALERT ${ }^{\circ}$ FA plus (aerobic) and FN plus (anaerobic) blood culture bottles were incubated in the BacT/ALERT ${ }^{\circ}$ 3D (before November 2016) or the $\mathrm{VIRTUO}^{\circ}$ System (bioMérieux, Inc. Marcy l'Etoile, France). Initial identification was performed on blood culture isolates cultured at $35^{\circ} \mathrm{C}$ in $\mathrm{CO}_{2}$ enriched atmosphere by MALDI-TOF MS (Bruker 
Biotyper; Bruker Daltonics, Bremen, Germany) using the MBT 6903 MSP Library, and for selected isolates with $16 \mathrm{~S}$ rRNA gene sequencing using the MicroSeq $50016 \mathrm{~S}$ rDNA PCR and Sequencing Kits (Applied Biosystems, Foster City, CA).

\section{MALDI-TOF MS- based typing}

MALDI-TOF MS-based typing was performed as described previously, with minor modifications [23]. In brief, fresh sub-cultured isolates were used and, after full protein extraction with formic acid, four spectra were generated for each isolate with a Microflex ${ }^{\mathrm{Tm}}$ MALDITOF (Bruker Daltonics). Using the flexAnalysis ${ }^{\text {Tn }}$ software version 3.4 (Bruker Daltonics) spectra were visually examined in the "overlaid" and "list view" mode for differentiating peaks among the eight isolates. Due to slow growth of the subcultures the incubation time was extended from $24 \mathrm{~h}$ to $48 \mathrm{~h}$ to yield more pronounced $\mathrm{m} / \mathrm{z}$ peaks. A peak list was generated with peaks with a signal intensity of $>1000$ arbitrary units; peaks with a signal-to-noise ratio $<10$ were only included in the analysis if they were visually clearly distinguishable from background level (Additional file 1: Figure S2). From the resulting binary peak list a distance matrix (dissimilarity structure) was computed (function dist) and a hierarchical cluster (function hclust) was generated in $\mathrm{R}$ [31]. Principal component analysis (PCA) was performed with MALDI Biotyper Compass Explorer 4.1 using standard settings.

\section{Genome-based typing}

Pulsed-field gel electrophoresis (PFGE): PFGE was performed with chromosomal DNA digested with Pme I as previously described [32].

WGS: All isolates were sequenced on an Illumina MiSeq, 2x300bp following Nextera XT library preparation. Data analysis was performed in CLC genomics Workbench v9.5.3. All samples were mapped against a de novo assembly of isolate $\mathrm{F}$, showing that mean sequencing coverage of all isolates was over 58x. A further within-cluster comparison was based on the mapping against the assembly of the genome of isolate B. Variants were called at $10 \mathrm{x}$ minimum coverage, 10 minimum count and $70 \%$ minimum frequency. Single nucleotide polymorphism (SNP) tree generation used a neighborjoining method: minimum coverage $10 \%$, minimum $\mathrm{z}-$ score 1.96, multi-nucleotide variants included. Digital DNA:DNA hybridization [33] was performed against all known Agrobacterium genomospecies [18] and confirmed using average nucleotide identity (ANI; http:// enve-omics.ce.gatech.edu/ani/). Mapping was also performed against plasmids pTi-SAKURA (accession number NC_002147.1) and pRi1724 (NC_002575.1), showing low $(<20 \%)$ fraction of plasmids covered at low $(<10 x)$ coverage. The datasets supporting the conclusions of this article are available in the European Nucleotide Archive repository, under Project number PRJEB34002 [https://www.ebi.ac.uk/ena/data/view/PRJEB34002] (Additional file 1: Table S2).

\section{Results \\ Epidemiological and clinical investigation for the time period 2011-2017}

In a two-week period between November 29 and December 7, 2015, we isolated $A$. radiobacter from blood cultures of two patients (Isolates A and B, =cluster I) admitted to the cardiology department. A close relation between the two isolates was suspected after rapid visual examination of their MALDI-TOF MS profiles (data not shown).

In October 2016 two additional patients presented with Agrobacterium sp. bloodstream infections within a two-week period (Isolates C and D; = cluster II) and two more cases occurred in January and February 2017 (Isolates $\mathrm{E}$ and $\mathrm{F}$; = cluster II). The isolates were identified with MALDI-TOF MS as Agrobacterium sp. with $A$. radiobacter as best match. These additional cases led to an extended epidemiological and clinical investigation encompassing all cases between 2011 and 2017 at Bern University Hospital. Two further cases (Isolates G and $\mathrm{H})$ were identified, resulting in a total of eight cases.

The clinical characteristics of the eight patients are provided in Tables 1 and 2 . The median age was 53 years (range 18-83 years), the majority were male (63\%). Three patients were admitted to medical wards, three to a surgical ward, one was treated in the emergency room and one in the radiology outpatient clinic.

All patients underwent a CT-scan with contrast media injection before developing bacteremia. The time interval between diagnostic radiology and positive blood culture varied with each cluster. For cluster I (Isolates $\mathrm{A}$ and $\mathrm{B}$ ) the median time-to-bacteremia was $81.3 \mathrm{~h}$ (range: $74.6-88.1 \mathrm{~h}$ ); for cluster II (Isolates C, D, $\mathrm{E}$ and F) $1.0 \mathrm{~h}$ (range: $40 \mathrm{~min}-59.4 \mathrm{~h}$ ); and for cases $\mathrm{G}$ and H 26 and 19 days, respectively. Two of eight case patients $(\mathrm{G}$ and $\mathrm{H})$ presented with a polymicrobial bloodstream infection. Six patients (75\%) presented with fever, the remaining two were afebrile. Three patients (37.5\%) presented with a central line-associated bloodstream infection (CLABSI), three (37.5\%) with transient bacteremia, one (12.5\%) with bloodstream infection and one $(12.5 \%)$ a catheter-related bloodstream infection. All patients were initially treated with intravenous antibiotics with variable duration (see Table 2 for details) and all patients were cured at the end of treatment without further infection-related complications. In all patients who had an intravascular device at the time of bacteremia, the device was removed. 
Table 1 Epidemiological data of eight Agrobacterium cases

\begin{tabular}{|c|c|c|c|c|c|c|c|c|c|c|c|}
\hline Patient & Age & Sex & $\begin{array}{l}\text { Clinical } \\
\text { service }\end{array}$ & $\begin{array}{l}\text { Date of hospital } \\
\text { admission }\end{array}$ & Cluster & $\begin{array}{l}\text { CT-scan } \\
\text { Room n }\end{array}$ & $\begin{array}{l}\text { Date of } \\
\mathrm{CT} \text { - } \\
\text { angiogram }\end{array}$ & $\begin{array}{l}\text { Date of } \\
\text { first } \\
\text { positive } \\
\text { BC }\end{array}$ & $\begin{array}{l}\Delta \mathrm{t} C \mathrm{CT}- \\
\text { positive } \mathrm{BC}\end{array}$ & Isolate & $\begin{array}{l}\text { Other invasive } \\
\text { procedures }\end{array}$ \\
\hline 1 & 47 & $M$ & Medicine & 23.08 .2011 & NA & CT1-R & 27.07 .11 & 23.08 .11 & $\begin{array}{l}26 \mathrm{~d} 23 \mathrm{~h} 44 \\
\mathrm{~m}\end{array}$ & $G$ & none \\
\hline 2 & 38 & $F$ & Oncology & 07.04.2013 & NA & CT2-ER & 12.04 .13 & 02.05 .13 & $\begin{array}{l}\text { 19d } 17 \text { h } 39 \\
\text { m }\end{array}$ & $\mathrm{H}$ & $\begin{array}{l}\text { Repeated self-injection of } \\
\text { sedatives }\end{array}$ \\
\hline 3 & 72 & M & Surgery & 26.11 .2015 & 1 & CT1-R & 26.11 .15 & 29.11 .15 & $3 \mathrm{~d} 2 \mathrm{~h} 34 \mathrm{~m}$ & A & TAVI, CA \\
\hline 4 & 83 & $\mathrm{~F}$ & Surgery & 01.12 .2015 & 1 & $\mathrm{CT} 1-\mathrm{R}$ & 03.12 .15 & 07.12 .15 & $3 \mathrm{~d} 16 \mathrm{~h} 6 \mathrm{~m}$ & B & $C A$ \\
\hline 5 & 18 & M & Medicine & 01.10 .2016 & 2 & CT2-ER & 01.10 .16 & 01.10 .16 & od $1 \mathrm{~h} 4 \mathrm{~m}$ & C & none \\
\hline 6 & 58 & M & $\begin{array}{l}\text { Emergency } \\
\text { Room }\end{array}$ & 12.10 .2016 & 2 & CT2-ER & 12.10 .16 & 12.10 .16 & Od $0 \mathrm{~h} 40 \mathrm{~m}$ & D & none \\
\hline 7 & 81 & M & Surgery & 10.01.2017 & 2 & CT2-ER & 21.01 .17 & 23.01 .17 & $2 \mathrm{~d} 11 \mathrm{~h} 22 \mathrm{~m}$ & E & Coronary bypass-surgery \\
\hline 8 & 19 & $\mathrm{~F}$ & Radiology & 24.02 .2017 & 2 & CT2-ER & 24.02 .17 & 24.02 .17 & Od $1 \mathrm{~h} 1 \mathrm{~m}$ & $\mathrm{~F}$ & none \\
\hline
\end{tabular}

Abbreviations: BC Blood culture, CA Coronary angiography, CT-R Computed Tomography-scan radiology (=CT Room 1), CT-ER Computed Tomography-scan emergency room, TAVI Transcatheter aortic valve replacement, Delta Time $(\Delta \mathrm{t}), h$ hour(s), $m$ minute(s), $d:$ day(s)

By means of a line list we identified a commonality for all cases in the form of the intravenous contrast medium injector in two Computer Tomography (CT) rooms (CT Exprés $^{\text {twa }}$, Debiotech S. A, Lausanne, Switzerland). For cases A and B we could not exclude a possible common source in the contrast media injector used in the same cardiac catheter laboratory (ACIST CVi ${ }^{\mathrm{m}}$, ACIST Medical System, Eden Prairie, MN, U.S.A.), despite negative microbiological testing (Additional file 1: Figure S1). Beside the epidemiological links, a structural infection risk was identified in the semi-open design of this device (Additional file 1: Figures. S3 and S4). Device contamination could also have been facilitated by construction works adjacent to the cardiac catheter laboratory. Apart from $\mathrm{A}$ and $\mathrm{B}$ no other patient underwent an invasive cardiology procedure; therefore, we excluded this device as possible common source for all cases. Environmental screening in both, CT scan room and cardiac catheter laboratory, was performed on the contrast media injector pump (including fixed and changeable parts) and contrast media solution. Environmental testing was also performed on plants present in the corridor of the radiology department. (Additional file 1: Table S1). None of the cultures revealed growth of Agrobacterium spp.

\section{MALDI-TOF MS-, PFGE- and WGS-based typing}

All strains were typed by MALDI-TOF MS, PFGE and WGS. MALDI-TOF MS-typing based on visual examination of the peak profiles and PCA confirmed two temporally related clusters and two outliers (Fig. 1a and Additional file 1: Figure S5): Cluster I (isolates A, B) occurred between November and December 2015 and cluster II (isolates C, D, E, F) between October 2016 and February 2017. According to the MALDI-TOF MS peak list the isolates within these clusters were identical, while a difference of eight $\mathrm{m} / z$ peaks was recorded to isolate $\mathrm{H}$ (Additional file 1: Table S3). Isolate $\mathrm{G}$ was unrelated, both chronologically and in terms of MALDI-TOF MS-typing results.

The dendrogram based on PFGE analysis was highly similar to the MALDI-TOF MS dendrogram in clearly delineating the two outbreak clusters I and II and the two separate isolates $\mathrm{G}$ and $\mathrm{H}$ (Additional file 1: Figure S6).

The WGS phylogenetic results further confirmed the clonality of the two clusters. The genomes of $\mathrm{A}$ and $\mathrm{B}$ differed by a single nucleotide polymorphism (SNP) (Fig. 1b). Digital DNA:DNA hybridization indicated that this cluster was most closely related to the $A$. radiobacter (= Agrobacterium genomovar G4) type strain LMG140 ${ }^{\mathrm{T}}$ (Additional file 1: Table S4). Cluster II consisted of isolates C, D, E and F, which all contained identical genomes, whereas isolate $\mathrm{H}$ shared only $89 \%$ of its genome with these four isolates, with the shared portion differing by over 2500 SNPs from the cluster (Fig. 1b), thereby validating the MALDI-TOF MS findings. Larger variable regions differing between these genomes include plasmids, phages, and integrative and conjugative elements / composite transposons. Digital DNA:DNA hybridization indicated that the strains in Cluster II, and isolate $\mathrm{H}$, were most closely related to Agrobacterium genomovar G3.

Isolate G, finally, was clearly separated from all other isolates by all typing methods. According to the WGS results it could be assigned to the species $A$. pusense (= Agrobacterium genomovar G2). None of the isolates were found to carry pTi or pRi plasmids.

\section{Discussion}

We report an outbreak investigation of eight cases of nosocomial Agrobacterium spp. bacteremia at a single tertiary referral center between 2011 and 2017. Environmental bacteria growing in blood cultures are usually 


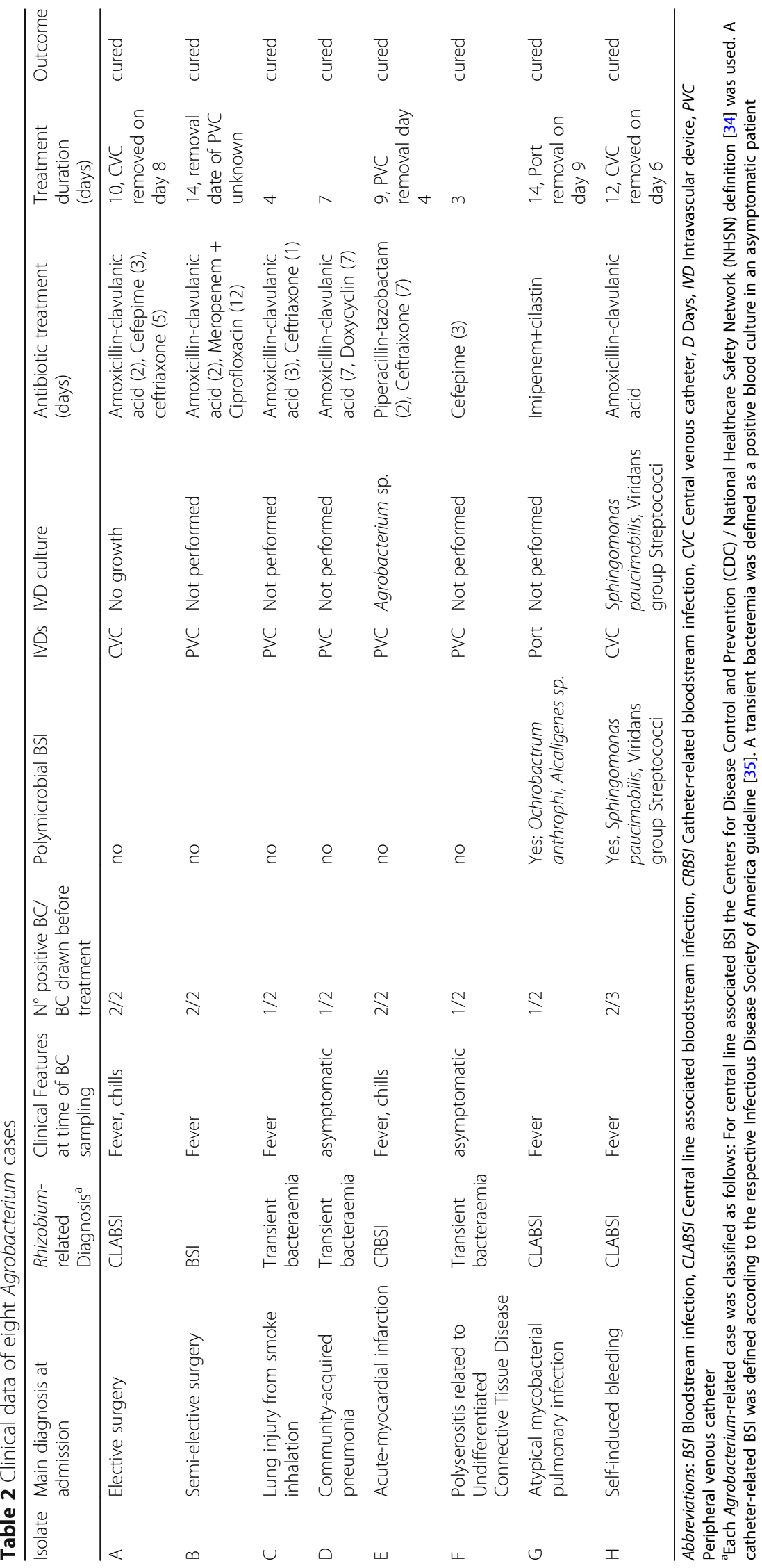




\section{A}

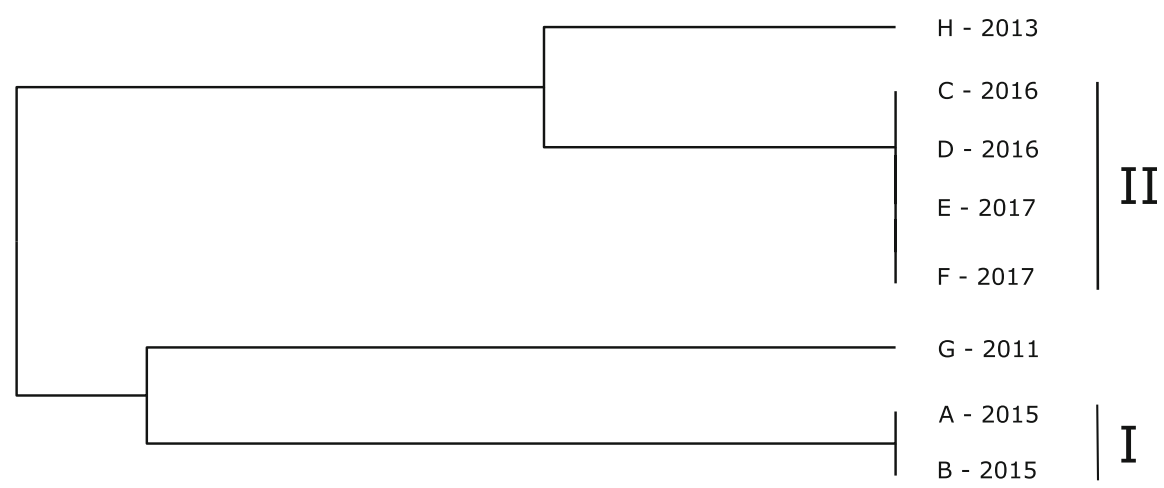

B

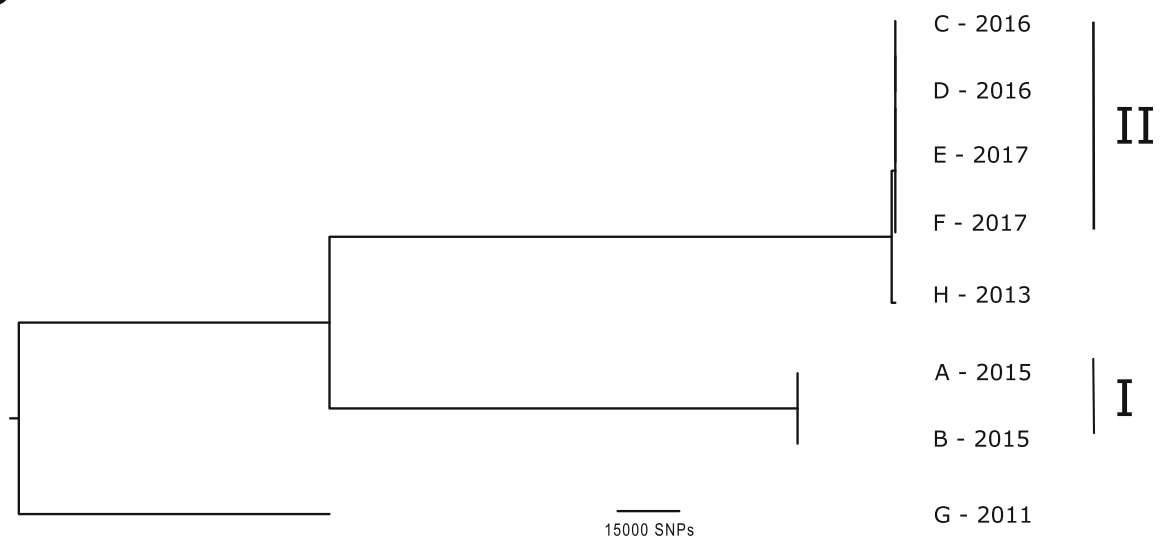

Fig. 1 a Hierarchical cluster based on observed differences in the MALDI-TOF peak profile of cultures grown for $48 \mathrm{~h}$. Indicated are the year of isolation for isolates A-H and the two outbreak clusters I and II. b SNP variant phylogenetic tree of sequenced isolates. The genome of isolate $\mathrm{F}$ was used as reference for the phylogeny. Scale bar indicates numbers of SNP differences between the isolates. Isolates $H, A / B$ and G map to only 89,76 and $77 \%$ of the reference genome, respectively

regarded as contaminants. Members of the genus Agrobacterium are, however, recognized as opportunistic human pathogens, particularly in intravascularly catheterized immunocompromised patients [5]. As their isolation from clinical specimen occurs relatively infrequently, temporal clustering of cases in our hospital triggered an outbreak investigation. Despite the fact that no Agrobacterium spp. were detected in the contrast medium itself, the epidemiological investigation identified the CT contrast application as the most probable common source of the outbreak. One conceivable transmission pathway is that the pathogen was transferred from the hands of staff to the device and then to the patient. However, the short times to the detection argue for a relatively high inoculum. This would speak for a previous replication in a medium. Since no such medium was found, we must admit that the final transmission mechanism remains unclear. We enforced hand hygiene precautions when handling the $\mathrm{CT}$ contrast medium injections and no further cases have since been detected at our institution.

A limitation of this study was the inability to microbiologically confirm either an environmental or a device contamination. Further, no prospective assessment of blood cultures of all patients undergoing CT injection was performed. This could have been reason for bias possibly, many more patients were affected but never recognized.

For cluster I the cardiac catheterization laboratory remained a suspected common source because of the semi-open design of the contrast medium injector that was found to pose an infectious risk, similar to the risk previously highlighted in other semi-open devices (e.g., syringes) [36]. The clinical outcome was favorable for all patients, as highlighted in other studies [8], irrespective of the use of different treatment regimens which followed the specific antibiogram. 
Most isolates described in previous reports on human Agrobacterium infections were identified with conventional methods which do not allow differentiation between the various genomovars. In a large study using multilocus sequence-based analysis, the majority of clinical isolates were found to belong to $A$. pusense (genomovar G2) which, consequently, was considered as human-associated genomovar [37].

In our case series only one of the eight isolates was assigned to $A$. pusense (Isolate G), whereas the outbreak clusters I and II consisted of $A$. radiobacter and genomovar G3 strains, respectively. The commercial MALDITOF MS database used for the identification of the clinical isolates contains 14 entries for $A$. radiobacter, one for $A$. rubi (in the database deposited as "Rhizobium radiobacter" and "Rhizobium rubi") and one for R. tropici. Using the Biotyper software, genomovar G2 and G4 isolates were identified with high confidence levels as " $R$. radiobacter" (score $>2.2$ ), while genomovar G3 isolates were identified merely to the genus level (score $>1.7$ to $<2.0$ for " $R$. radiobacter").

Using MALDI-TOF MS-typing via visual examination of the peak profiles, the three genomovars clearly fell into three clusters (Fig. 1a), indicating that an identification of individual genomovars based on MS spectra could be achieved using a well-characterized reference database. Comparison of the spectra with a database generated on the base of putatively ascribed ribosomal protein masses extracted from genomic data [38] accordingly allowed a genomovar assignment for all eight isolates (Additional file 1: Table S5). An advanced routine identification method for Agrobacterium isolates would in turn lead to a better understanding of the role and prevalence of different Agrobacterium genomovars in human infections.

While WGS is currently replacing PFGE as the "gold standard" for molecular epidemiological typing, a growing number of reports demonstrate the utility of MALDITOF MS as a rapid, relatively inexpensive method for outbreak investigation. One concern about this method is the current lack of interpretation guidelines for strain relatedness [39] and its lower resolution due to technical limitations. As it is mostly based on the proteome phenotype, which in turn is influenced by horizontal gene transfer of accessory or even ribosomal genes, it does not necessarily reflect evolutionary relatedness. The isolates in our analysis belong to a genus with a high degree of diversity, including in its accessory gene repertoire [40]. The individual genomovars were thus clearly separated by more than 20 peak differences. Furthermore, discrimination of strains within a genomovar was achieved. Isolate $\mathrm{H}$ was isolated more than 3 years before the isolates in cluster II but, interestingly, the patient with this isolate underwent contrast medium injection in the same CT room. The isolate could clearly be distinguished by a difference of eight MALDI-TOF MS peaks from the other genomovar G3 isolates, which corresponded to a divergence of 2671 SNPs and $11 \%$ of the genome in WGS-based typing.

In this report we highlight the use of MALDI-TOF MS as a readily available typing method to recognize hospital outbreaks more rapidly than genome-based methods such as PFGE or WGS. While the current "gold-standard" PFGE or WGS require several days or perhaps longer for rare pathogens, MALDI-TOF MSbased typing can be achieved within a single day. This method may prove to be extremely valuable in ongoing outbreak situations with gram-negative pathogens: elucidating quickly whether newly identified isolates belong to the outbreak cluster or not could greatly help to resolve an outbreak.

\section{Conclusion}

In conclusion, we report recurrent clusters of Agrobacterium spp. bacteremia likely acquired during CT contrast medium injection in two different $\mathrm{CT}$ rooms. Also, we highlight the use of MALDI-TOF MS-based typing as a method for rapid identification of outbreak clusters due to rare pathogens.

\section{Additional file}

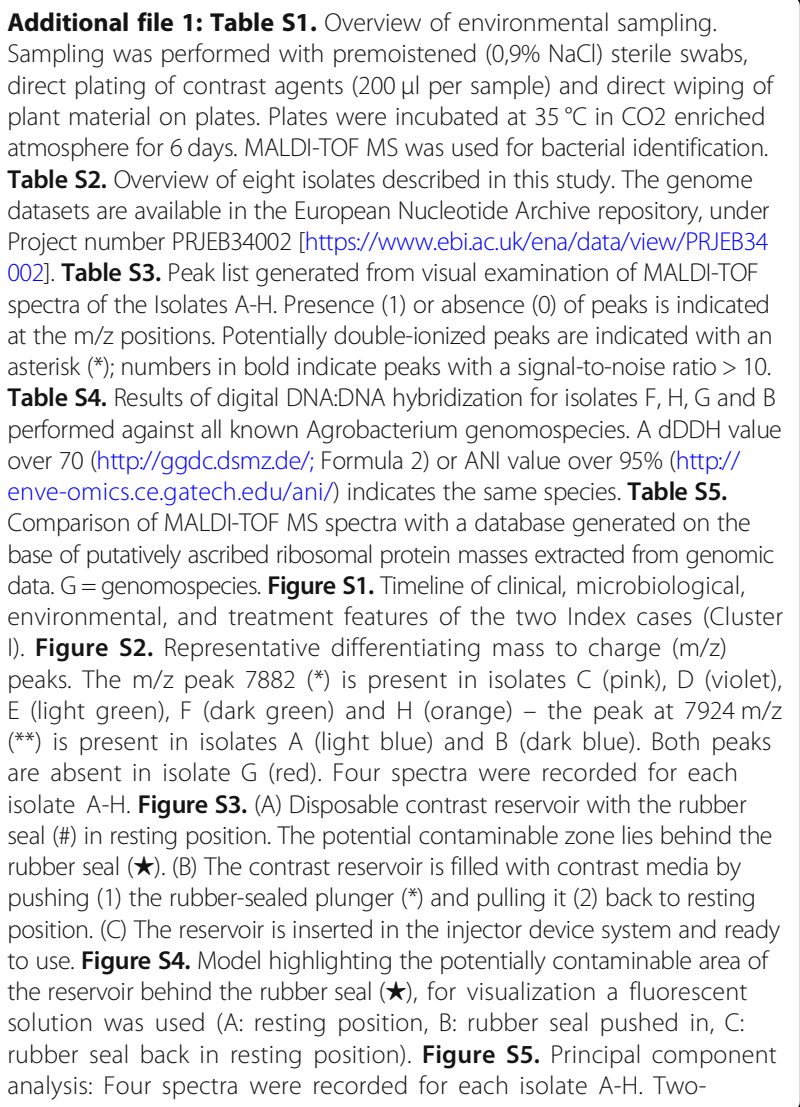


dimensional plot of the first two principal components indicating the two clusters I (isolates A and B) and II (isolates C, D, E and F) and the "unrelated" isolates $\mathrm{G}$ and $\mathrm{H}$. Figure S6. PFGE based typing of isolates A-H. The scale indicates the degree of similarity (\%) as calculated by dice correlation analysis.

\section{Acknowledgments}

We would like to thank M. Laguardia, W. Steiger, N. Buetti MD, A. Künzli MD, members of the Infection Control Team, for their assistance and support during the investigation. We appreciate Christoph Hauser, MD, Bern University Hospital for critical discussions on the topic. Lastly, we thank Magdalena Schneider, Clarisse Straub, Elisabeth Schultheiss, Christine Kiessling, and Rosamaria Vesco, all at University Hospital Basel, for excellent technical assistance in PFGE and WGS based typing.

\section{Author's contributions}

$E L, R S, J M, C C, S D, L R$ and DO conducted epidemiological and clinical investigations. CC, VP, AE and SD performed laboratory analysis and MALDITOF MS based typing, HS and AE performed genome-based typing. All authors contributed to the manuscript and agreed on the final version for submission. All authors read and approved the final manuscript.

\section{Funding}

None.

\section{Availability of data and materials}

All data generated or analysed during this study are included in this article, its supplementary information files or are available from the corresponding author on reasonable request.

\section{Ethics approval}

According to current Swiss law, outbreak investigation is considered to be part of quality assurance and is therefore exempt from ethical consent.

\section{Consent for publication}

Not applicable.

\section{Competing interests}

The authors declare that they have no competing interests.

\section{Author details}

'Institute for Infectious Diseases, University of Bern, Friedbühlstrasse 51, 3001 Bern, Switzerland. ${ }^{2}$ Department of Infectious Diseases, Bern University Hospital, University of Bern, Freiburgstrasse, 3001 Bern, Switzerland. ${ }^{3}$ Division of Clinical Microbiology, University Hospital Basel, Basel, Switzerland. ${ }^{4}$ Applied Microbiology Research, Department of Biomedicine, University of Basel, Basel, Switzerland. ${ }^{5}$ Department of Cardiology, Bern University Hospital, University of Bern, Bern, Switzerland. ${ }^{6}$ Department of Radiology, Bern University Hospital, University of Bern, Bern, Switzerland. 'Mabritec AG, Riehen, Switzerland.

\section{Received: 12 July 2019 Accepted: 1 October 2019}

\section{Published online: 04 November 2019}

\section{References}

1. Mastroianni A, Coronado O, Nanetti A, Manfredi R, Chiodo F. Agrobacterium radiobacter pneumonia in a patient with HIV infection. Eur J Clin Microbiol Infect Dis. 1996;15:960-3.

2. Detrait $M, D^{\prime}$ Hondt $L$, André $M$, Lonchay $C$, Holemans $X$, Maton JP, et al. Agrobacterium radiobacter bacteremia in oncologic and geriatric patients: presentation of two cases and review of the literature. Int J Infect Dis. 2008; 12:e7-10.

3. Kaselitz TB, Hariadi NI, LiPuma JJ, Weinberg JB. Rhizobium radiobacter bacteremia in a neonate. Infection. 2012;40:437-9.

4. Chen C-Y, Hansen KS, Hansen LK. Rhizobium radiobacter as an opportunistic pathogen in central venous catheter-associated bloodstream infection: case report and review. J Hosp Infect. 2008:68:203-7.

5. Paphitou NI, Rolston KVI. Catheter-related bacteremia caused by agrobacterium radiobacter in a cancer patient: case report and literature review. Infection. 2003;31:421-4.
6. Pascart T, Ducoulombier V, Budzik JF, Dehecq E, Houvenagel E. Spondylodiscitis due to rhizobium radiobacter. Médecine Mal Infect. 2015: 45:181-2.

7. Moreau-Gaudry V, Chiquet C, Boisset S, Croize J, Benito Y, Cornut PL, et al. Three cases of post-cataract surgery Endophthalmitis due to rhizobium (agrobacterium) radiobacter. J Clin Microbiol. 2012;50:1487-90.

8. Lai C-C, Teng L-J, Hsueh P-R, Yuan A, Tsai K-C, Tang J-L, et al. Clinical and microbiological characteristics of rhizobium radiobacter infections. Clin Infect Dis. 2004;38:149-53.

9. Guerra NC, Nobre A, Cravino J. Native mitral valve endocarditis due to rhizobium Radiobacter - first case report. Rev Port Cir Cardio-Torácica E Vasc. 2013;20:203-5.

10. Plotkin GR. Agrobacterium radiobacter prosthetic valve endocarditis. Ann Intern Med. 1980;93:839-40.

11. Giammanco GM, Pignato $S$, Santangelo C, Grimont PAD, Grimont F, Giammanco G. Molecular typing of agrobacterium species isolates from catheter-related bloodstream infections. Infect Control Hosp Epidemiol. 2004;25:885-7.

12. Rogues AM, Sarlangue J, de Barbeyrac B, Doerman HP, Helmer C, Labadie JC, et al. Agrobacterium radiobacter as a cause of pseudobacteremia. Infect Control Hosp Epidemiol. 1999;20:345-7.

13. Pillonetto M, Arend L, Gomes SMT, Oliveira MAA, Timm LN, Martins AF, et al. Molecular investigation of isolates from a multistate polymicrobial outbreak associated with contaminated total parenteral nutrition in Brazil. BMC Infect Dis. 2018;18:397.

14. Farrand SK, Van Berkum PB, Oger P. Agrobacterium is a definable genus of the family Rhizobiaceae. Int J Syst Evol Microbiol. 2003;53:1681-7.

15. Young JM, Kuykendall LD, Martínez-Romero E, Kerr A, Sawada H. Classification and nomenclature of agrobacterium and rhizobium. Int I Syst Evol Microbiol. 2003:53:1689-95.

16. Ormeño-Orrillo E, Servín-Garcidueñas LE, Rogel MA, González V, Peralta H, Mora J, et al. Taxonomy of rhizobia and agrobacteria from the Rhizobiaceae family in light of genomics. Syst Appl Microbiol. 2015;38:287-91.

17. de Lajudie PM, Young JPW. International committee on systematics of prokaryotes Subcommittee for the Taxonomy of rhizobium and agrobacterium minutes of the meeting, Budapest, 25 august 2016. Int I Syst Evol Microbiol. 2017;67:2485-94.

18. Mousavi SA, Willems A, Nesme X, de Lajudie P, Lindström K. Revised phylogeny of Rhizobiaceae: proposal of the delineation of Pararhizobium gen. Nov., and 13 new species combinations. Syst App Microbiol. 2015;38:84-90.

19. Tindall BJ. Agrobacterium radiobacter (Beijerinck and van Delden 1902) conn 1942 has priority over agrobacterium tumefaciens (smith and Townsend 1907) conn 1942 when the two are treated as members of the same species based on the principle of priority and rule 23a, note 1 as applied to the corresponding specific epithets. Opinion 94. Judicial Commission of the International Committee on systematics of prokaryotes. Int J Syst Evol Microbiol. 2014;64:3590-2.

20. Mafakheri H, Taghavi SM, Puławska J, de Lajudie P, Lassalle F, Osdaghi E. Two Novel Genomospecies in the Agrobacterium tumefaciens Species Complex Associated with Rose Crown Gall. Phytopathology. 2019. https:// doi.org/10.1094/PHYTO-05-19-0178-R

21. Sabat AJ, Budimir A, Nashev D, Sá-Leão $R, J m \vee D$, Laurent F, et al. Overview of molecular typing methods for outbreak detection and epidemiological surveillance. Euro Surveill. 2013;18:20380.

22. Rossen JWA, Friedrich AW, Moran-Gilad J, ESCMID study Group for Genomic and Molecular Diagnostics (ESGMD). Practical issues in implementing whole-genome-sequencing in routine diagnostic microbiology. Clin Microbiol Infect. 2018;24:355-60.

23. Egli A, Tschudin-Sutter S, Oberle M, Goldenberger D, Frei R, Widmer AF. Matrixassisted laser desorption/ionization time of flight mass-spectrometry (MALDITOF MS) based typing of extended-spectrum $\beta$-lactamase producing E. coli--a novel tool for real-time outbreak investigation. PloS One. 2015;10:e0120624.

24. Schlebusch S, Price GR, Hinds S, Nourse C, Schooneveldt JM, Tilse MH, et al. First outbreak of PVL-positive nonmultiresistant MRSA in a neonatal ICU in Australia: comparison of MALDI-TOF and SNP-plus-binary gene typing. Eur J Clin Microbiol Infect Dis. 2010;29:1311-4.

25. Verroken A, Bauraing C, Deplano A, Bogaerts P, Huang D, Wauters G, et al. Epidemiological investigation of a nosocomial outbreak of multidrugresistant Corynebacterium striatum at one Belgian university hospital. Clin Microbiol Infect. 2014;20:44-50. 
26. Khennouchi NC, Loucif L, Boutefnouchet N, Allag H, Rolain J-M. MALDI-TOF MS as a tool to detect a nosocomial outbreak of extended-Spectrum- $\beta$ lactamase- and ArmA methyltransferase-producing Enterobacter cloacae clinical isolates in Algeria. Antimicrob Agents Chemother. 2015;59:6477-83.

27. Mencacci A, Monari C, Leli C, Merlini L, De Carolis E, Vella A, et al. Typing of nosocomial outbreaks of Acinetobacter baumannii by use of matrix-assisted laser desorption ionization-time of flight mass spectrometry. J Clin Microbiol. 2013;51:603-6.

28. Oberle M, Wohlwend N, Jonas D, Maurer FP, Jost G, Tschudin-Sutter S, et al. The technical and biological reproducibility of matrix-assisted laser desorption ionization-time of flight mass spectrometry (MALDI-TOF MS) based typing: employment of bioinformatics in a multicenter study. PLoS One. 2016;11:e0164260.

29. Østergaard C, Hansen SGK, Møller JK. Rapid first-line discrimination of methicillin resistant Staphylococcus aureus strains using MALDI-TOF MS. Int J Med Microbiol. 2015;305:838-47.

30. Lasch P, Fleige C, Stämmler M, Layer F, Nübel U, Witte W, et al. Insufficient discriminatory power of MALDI-TOF mass spectrometry for typing of enterococcus faecium and Staphylococcus aureus isolates. J Microbiol Methods. 2014;100:58-69.

31. R Core Team (2014). R: a language and environment for statistical computing. R Foundation for Statistical Computing.

32. Strandén A, Frei R, Widmer AF. Molecular typing of methicillin-resistant Staphylococcus aureus: can PCR replace pulsed-field gel electrophoresis? J Clin Microbiol. 2003;41:3181-6.

33. Auch AF, von Jan M, Klenk H-P, Göker M. Digital DNA-DNA hybridization for microbial species delineation by means of genome-to-genome sequence comparison. Stand Genomic Sci. 2010;2:117-34.

34. Centers for Disease Control (CDC)/Healthcare Infection Control Practices Advisory Committee (HICPAC). Bloodstream Infection Event (Central LineAssopcciated Bloodstrea Infection and Non-central line-associated Bloodstream Infection). 2015 [cited 2015 Dec 3]. Available from: http://www. cdc.gov/nhsn

35. Mermel LA, Allon M, Bouza E, Craven DE, Flynn P, O'Grady NP, et al. Clinical practice guidelines for the diagnosis and management of intravascular catheter-related infection: 2009 update by the Infectious Diseases Society of America. Clin Infect Dis. 2009:49:1-45.

36. Clack L, Kuster SP, Giger H, Giuliani F, Sax H. Low-hanging fruit for human factors design in infection prevention--still too high to reach? Am J Infect Control. 2014;42:679-81.

37. Aujoulat F, Marchandin H, Zorgniotti I, Masnou A, Jumas-Bilak E. Rhizobium pusense is the main human pathogen in the genus Agrobacterium/ Rhizobium. Clin Microbiol Infect. 2015;21:472.e1-5.

38. Ziegler D, Pothier JF, Ardley J, Fossou RK, Pflüger V, de Meyer S, et al. Ribosomal protein biomarkers provide root nodule bacterial identification by MALDI-TOF MS. Appl Microbiol Biotechnol. 2015;99:5547-62.

39. Spinali S, van Belkum A, Goering RV, Girard V, Welker M, Van Nuenen M, et al. Microbial typing by matrix-assisted laser desorption ionization-time of flight mass spectrometry: do we need guidance for data interpretation? J Clin Microbiol. 2015:53:760-5.

40. Lassalle F, Planel R, Penel S, Chapulliot D, Barbe V, Dubost A, et al. Ancestral genome estimation reveals the history of ecological diversification in agrobacterium. Genome Biol Evol. 2017;9:3413-31.

\section{Publisher's Note}

Springer Nature remains neutral with regard to jurisdictional claims in published maps and institutional affiliations.

Ready to submit your research? Choose BMC and benefit from:

- fast, convenient online submission

- thorough peer review by experienced researchers in your field

- rapid publication on acceptance

- support for research data, including large and complex data types

- gold Open Access which fosters wider collaboration and increased citations

- maximum visibility for your research: over $100 \mathrm{M}$ website views per year

At BMC, research is always in progress.

Learn more biomedcentral.com/submissions 